PROCEEDINGS OF THE

AMERICAN MATHEMATICAL SOCIETY

Volume 133, Number 9, Pages 2605-2608

S 0002-9939(05)07934-7

Article electronically published on March 31, 2005

\title{
A NOTE ON DE BRANGES' WEIGHTS
}

\author{
ARCADII Z. GRINSHPAN AND ANATOLII GRINSHPAN
}

(Communicated by Juha M. Heinonen)

\begin{abstract}
We discuss the de Branges weight systems and give a counterexample uncovering pitfalls.
\end{abstract}

\section{Preliminaries}

For a positive integer $n$ and $t \geq 0$, a de Branges weight system is a system of nonincreasing functions $\sigma_{m}(t)$ satisfying the differential recurrence

$$
\sigma_{m}-\sigma_{m+1}=-\frac{\dot{\sigma}_{m}}{m}-\frac{\dot{\sigma}_{m+1}}{m+1}, \quad m=1, \ldots, n, \quad \sigma_{n+1} \equiv 0 .
$$

The weight system defined by the initial conditions $\sigma_{m}(0)=n+1-m$ plays a key part in de Branges' famous proof of the inequality

$$
\sum_{m=1}^{n}(n+1-m)\left(m\left|c_{m}\right|^{2}-4 / m\right) \leq 0
$$

for the logarithmic coefficients $c_{m}$ of normalized univalent functions, conjectured by Milin and implying the Bieberbach conjecture 1] (results, history and bibliography can be found in [2, 4]).

In fact, de Branges' theorem contains the inequality

$$
\sum_{m=1}^{n} x_{m}\left(m\left|c_{m}\right|^{2}-4 / m\right) \leq 0
$$

for any $x_{m}$ that are the initial values $\sigma_{m}(0)$ of nonincreasing weights $\sigma_{m}$. An explicit or asymptotic knowledge of numbers $x_{1}, \ldots, x_{n}$ such that (2) holds potentially opens the door to other interesting estimates. Not all of these tuples arise as initial values of the nonincreasing solutions of (1), that is via de Branges' method, and their description is unknown. However, a complete description of all nonincreasing solutions of (11) is given in [3] in the framework of a more general setting.

Because of certain implications, our attention has recently been drawn to [6] which claims to obtain (2), by de Branges' method, for any choice of the initial

Received by the editors January 6, 2004.

2000 Mathematics Subject Classification. Primary 30C50, 30C55, 30C75.

Key words and phrases. Univalent functions, de Branges' weight systems, associated Legendre functions.

(C)2005 American Mathematical Society 
conditions satisfying

$$
-x_{m}+2 \sum_{k=m}^{n}(-1)^{k-m} x_{k} \geq 0, \quad m=1, \ldots, n .
$$

The purpose of this note is to explain why neither the method nor the result of $[\underline{6}]$ is correct.

Let $\mathbb{D}=\{z:|z|<1\}$ be the open unit disk. We will denote by $\{f\}_{m}$ the coefficient of $z^{m}$ in a power series $f$. The class $S$ is formed by all functions $f$ that are analytic and univalent in $\mathbb{D}$ and normalized by $f(0)=0, f^{\prime}(0)=1$. The logarithmic coefficients of $f \in S$ are the coefficients of $\log (f(z) / z)$. The Koebe function

$$
K(z)=\frac{z}{(1-z)^{2}}
$$

is in $S$. It maps $\mathbb{D}$ onto the plane slit along $\left(-\infty,-\frac{1}{4}\right]$. For $t \geq 0$, the Pick function $P(z, t)$ is defined implicitly by

$$
K(P(z, t))=e^{-t} K(z) .
$$

It is evident that $P(z, 0)=z$. For $t>0$,

$$
P(z, t)=e^{-t} z+2 e^{-t}\left(1-e^{-t}\right) z^{2}+e^{-t}\left(3-8 e^{-t}+5 e^{-2 t}\right) z^{3}+\ldots
$$

is a univalent map of $\mathbb{D}$ onto $\mathbb{D}$ cut along the real axis from -1 to the point $1+2\left(\sqrt{e^{2 t}-e^{t}}-e^{t}\right)$. For each $m \geq 1$, the coefficient $\{P(z, t)\}_{m}$ is a polynomial in $e^{-t}$ of degree $m$. A relevant property of the Pick function is given by the differential equation

$$
\dot{P}=-P \frac{1-P}{1+P}
$$

\section{The Weight SYSTEM}

Every set of solutions of (11) is uniquely determined by the initial conditions and can be written in terms of the coefficients of powers of the Pick function. Indeed, by (4) $P^{m}(z, t)$ satisfies the $m$-th equation of $(1), 1 \leq m \leq n$. Therefore

$$
\sigma_{m}=\sum_{k=m}^{n} x_{k}\left\{P^{m}\right\}_{k}, \quad m=1, \ldots, n,
$$

is the general solution of (11). One can also write the general solution in terms of the special functions. The most general nonincreasing solutions of (1) are determined by the initial data

$$
\sigma_{m}(0)=\sum_{k=m}^{n} a_{k} \beta_{m k}, \quad m=1, \ldots, n,
$$

where $\beta_{m k}$ are defined by means of the associated Legendre functions $P_{m k}$,

$$
\beta_{m k}=2 m \frac{(k-m) !}{(k+m) !} \int_{0}^{1} P_{m k}^{2}(x) \frac{x d x}{1-x^{2}},
$$

and $a_{k}$ are arbitrary nonnegative numbers [3]. In particular, the last three betas for each $m$ are as follows:

$$
\beta_{m m}=\frac{1}{4^{m}}\left(\begin{array}{c}
2 m \\
m
\end{array}\right), \quad \beta_{m-1 m}=\frac{2}{4^{m}}\left(\begin{array}{c}
2 m \\
m
\end{array}\right), \quad \beta_{m-2 m}=\frac{1}{4^{m}} \frac{5 m-2}{2 m-1}\left(\begin{array}{c}
2 m \\
m
\end{array}\right)
$$


Equations (1) can be written in the form

$$
\begin{aligned}
-\frac{\dot{\sigma}_{m}}{m} & =-\sigma_{m}+2 \sum_{k=m}^{n}(-1)^{k-m} \sigma_{k} \\
& =\sum_{k=m}^{n}(-1)^{k-m}\left(\sigma_{k}-\sigma_{k+1}\right), \quad m=1, \ldots, n .
\end{aligned}
$$

Hence a necessary and sufficient condition for the weights $\sigma_{m}$ to be nonincreasing is given by

$$
\sum_{k=m}^{n}(-1)^{k-m}\left(\sigma_{k}-\sigma_{k+1}\right) \geq 0, \quad m=1, \ldots, n .
$$

By adding these inequalities inductively we obtain a simple necessary condition

$$
\sigma_{1} \geq \cdots \geq \sigma_{n-1} \geq 2 \sigma_{n} \geq 0 .
$$

Obviously, (8) reduces to (3) for $t=0$. However, (3) by itself does not guarantee the nonincreasing behavior of weights. To see this, let $x_{1}=2, x_{2}=2, x_{3}=1$, and let the remaining $x_{k}$ be zero $(n \geq 3)$ in (5). Then

$$
\sigma_{1}=e^{-t}\left(9-12 e^{-t}+5 e^{-2 t}\right)
$$

is not monotone. Worse, one can check using (7) that for every $n \geq 3$ the initial data of the form $(\ldots, 2,2,1)$ forces $a_{n-2}$ in (6) to be negative, which contradicts monotonicity. So even if (2) is true in this case, de Branges' method cannot be applied to prove it.

\section{A counterexample}

We now show that merely under assumption (3) the inequality (2) can fail on $S$. For each $t,(8)$ describes a half-cone of vectors $\left(\sigma_{1}, \ldots, \sigma_{n}\right)$ in $\mathbb{R}^{n}$. It is generated by the linear combinations of vectors

$$
(1,0,0, \ldots, 0),(2,1,0, \ldots, 0), \ldots,(2,2, \ldots, 2,1)
$$

with nonnegative coefficients. Hence the conclusion of [6] is correct if and only if (21) holds for the initial data of the form

$$
(2, \ldots, 2,1,0, \ldots, 0) \text {. }
$$

To show that (21) actually fails for vectors $(2,2,1,0, \ldots, 0)$, we use a construction of the Schaeffer-Spencer type [5]; see also [2, 4] for details and references.

For $-1 \leq \varepsilon \leq 1$, the function

$$
g(z)=z(1-z)^{-1-\varepsilon}(1+z)^{-1+\varepsilon}, \quad z \in \mathbb{D},
$$

is starlike and belongs to $S$. Given $0<t \leq 1$, define $w(z, t)$ by the equation

$$
g(w(z, t))=\operatorname{tg}(z) .
$$

Note that $w(z, t)$ is a univalent map of $\mathbb{D}$ into itself. Furthermore, let

$$
F(z, t)=\frac{w(z, t)}{t(1-w(z, t))^{2}} .
$$

Clearly, $F(z, t) \in S$ for every admissible pair of parameters $\varepsilon$ and $t$. Write

$$
\log \frac{F(z, t)}{z}=c_{1} z+c_{2} z^{2}+c_{3} z^{3}+\ldots
$$


A routine calculation gives

$$
\begin{aligned}
& c_{1}=2(1-(1-\varepsilon)(1-t)), \\
& c_{2}=1+4 \varepsilon t(1-\varepsilon)(1-t), \\
& c_{3}=\frac{2}{3}+2(1-\varepsilon)(1-t)\left(2 \varepsilon^{2} t(1-3 t)+\frac{1}{3}(2 t(1+t)-1)\right) .
\end{aligned}
$$

It is now easy to see that the functional

$$
\begin{aligned}
J(F) & =2\left(\left|c_{1}\right|^{2}-4\right)+2\left(2\left|c_{2}\right|^{2}-4 / 2\right)+\left(3\left|c_{3}\right|^{2}-4 / 3\right) \\
& =2\left|c_{1}\right|^{2}+4\left|c_{2}\right|^{2}+3\left|c_{3}\right|^{2}-40 / 3
\end{aligned}
$$

can assume positive values. In fact, the pairs $(\varepsilon, t)$ close to $(.65, .85)$ result in the values of $J$ which are slightly greater than .002 . Thus the result of [6] is false for every $n \geq 3$.

\section{REFERENCES}

[1] L. de Branges, A proof of the Bieberbach conjecture, Acta Math. 154 (1985), no. 1-2, 137-152. MR0772434 (86h:30026)

[2] P. Duren, Univalent functions, Springer-Verlag, New York, 1983. MR0708494 (85j:30034)

[3] A. Grinshpan, Monotone functions of certain motions, preprint, Cal Tech, 2003.

[4] A. Z. Grinshpan, Logarithmic geometry, exponentiation, and coefficient bounds in the theory of univalent functions and nonoverlapping domains, Handbook of Complex Analysis: Geometric Function Theory, V. 1 (Ed. by R. Kühnau), 273-332, North-Holland, Amsterdam, 2002. MR1966197 (2003m:30041)

[5] A.C. Schaeffer and D.C. Spencer, The coefficients of schlicht functions, Duke Math. J. 10 (1943), 611-635. MR0009631 (5:175i)

[6] Xie Ming-Qin, A generalization of the de Branges theorem, Proc. of the AMS 125 (1997), no. 12, 3605-3611. MR1415355|(98b:30018)

Department of Mathematics, University of South Florida, Tampa, Florida 33620

E-mail address: azg@math.usf.edu

Mathematics 253-37, California Institute of Technology, Pasadena, California 91125 Current address: Department of Mathematics, Oklahoma State University, Stillwater, Oklahoma 74078-1058

E-mail address: agrinsh@math.okstate.edu 